\section{Pacific Northwest}

National Laboratory

Operated by Battelle for the

U.S. Department of Energy

\title{
Construction and Testing of a Low- Power Cryostat for MARS
}

\author{
CE Aalseth \\ JA Caggiano \\ JE Fast \\ AR Day \\ ES Fuller
}

September 2007

Prepared for the U.S. Department of Energy

under Contract DE-AC05-76RL01830 


\section{Construction and Testing of a Low Power Cryostat for MARS}
C. E. Aalseth
E. S. Fuller
J. A. Caggiano
A. R. Day
J. E. Fast

September 2007

Prepared for

the U.S. Department of Energy

under Contract DE-AC05-76RL01830

Pacific Northwest National Laboratory

Richland, Washington 99352 


\begin{abstract}
A low-power cryostat was designed and built for the Multi-sensor Airborne Radiation Survey (MARS) project for the purpose of housing a close-packed high-purity germanium (HPGe) detector array of 14 HPGe detectors. The power consumption of the cold mass in the cryostat was measured to be 4.07(11) watts, sufficient for 5.5 days of continuous operation using only 8 liters of liquid nitrogen. Temperatures throughout the cryostat were measured by platinum resistance temperature detectors. These measurements were used to determine the emissivity of the copper used in the floating radiation shield and outer cryostat wall, which was constructed using chemically cleaned and passivated copper metal. Using a PNNL-developed passivation process, an emissivity of $2.5(3) \%$ was achieved for copper.
\end{abstract}





\section{Glossary}

ERSS Environmental Radiation Sensor System

HPGe high-purity germanium

LN liquid nitrogen

MARS Multi-sensor Airborne Radiation Survey

MLI multi-layered insulation

NIM Nuclear Instrumentation Module

PCTFE polychlorotetrafluoroethylene

RTDs resistance temperature detectors

PNNL Pacific Northwest National Laboratory 



\section{Contents}

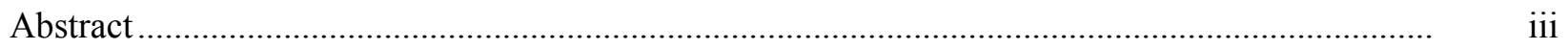

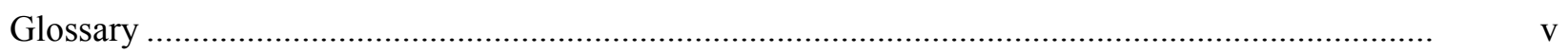

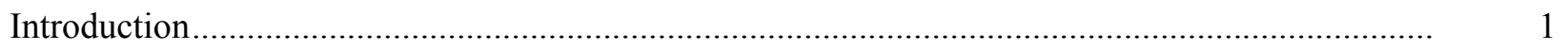

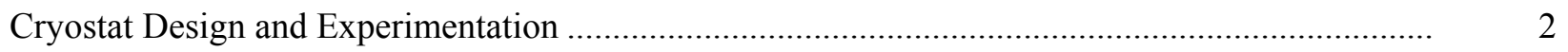

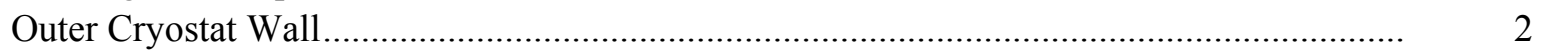

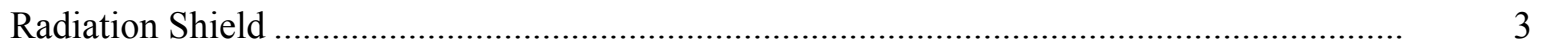

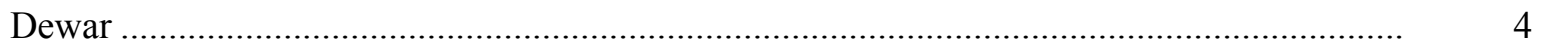

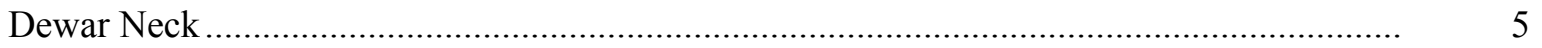

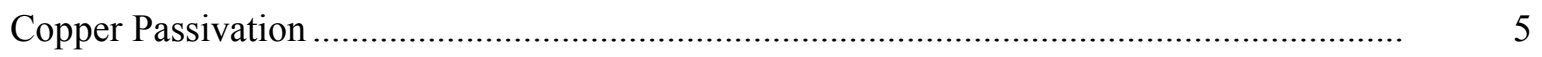

Temperature Measurement Apparatus....................................................................... 5

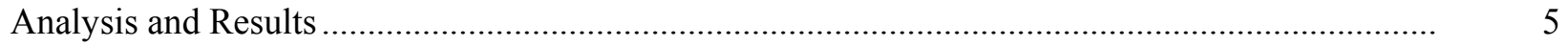

Heat Load Sources.................................................................................................. 6

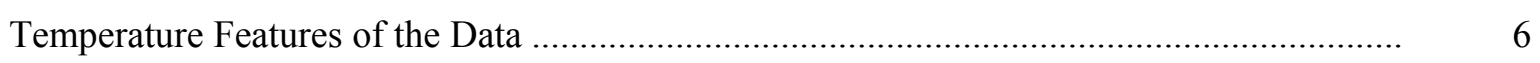

Emissivity Measurements .........................................................................................

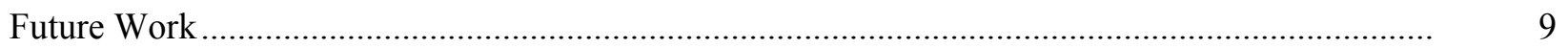

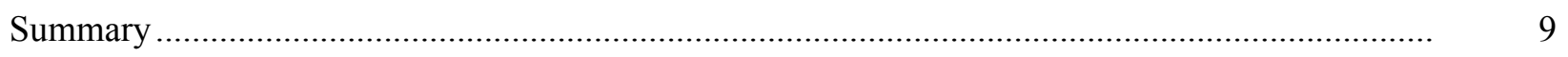

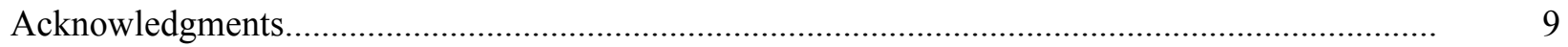

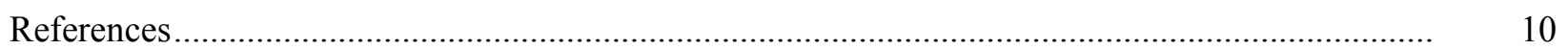




\section{Figures}

1. Schematic of the Cryostat as Tested in the Lowest Power Configuration ............................... 3

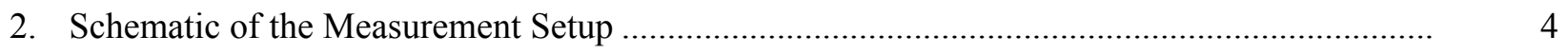

3. Temperature Profile of the Dewar and Radiation Shield During One of the Tests .................... 7

4. Measured Equilibrium Temperatures with a Full Loading of LN ......................................... 7

5. Instantaneous Power vs. Elapsed Time ….................................................................... 8 


\section{Introduction}

The primary objective of the Multiple Airborne Radiation Sensor (MARS) project is to develop a long-standoff gamma radiation detector for deployment on a helicopter or other flying vehicle with the following characteristics:

- high resolution

- highly efficient

- low power consumption

- low liquid nitrogen (LN) consumption

- compact

- relatively light (of order $100 \mathrm{lbs}$ )

- directional.

The goal of the MARS project is to not only duplicate the functionality of the Environmental Radiation Sensor System (ERSS) (Lepel et al. 1998) but to improve the system dramatically by modernizing the design.

The ERSS was a fairly compact system of many high-purity germanium (HPGe) detectors placed in a "pod" that could be mounted on a helicopter and was used to detect radiation from relatively large standoff distances. The system as outlined in the paper had several operational limitations. The hold time of the LN was only four hours, and each module weighed $311 \mathrm{~kg}$, not including two full-height racks of Nuclear Instrumentation Module (NIM) electronics; the whole system weighed $817 \mathrm{~kg}$. Given the $\sim 180$ liters of LN, this 45 -liters/hr consumption corresponds to roughly one kilowatt of power being transferred to the cold mass.

The primary objective of the current effort was to design and test a cryostat that uses far less LN and minimizes thermal load on the cold mass. The improved design incorporates floating infrared-radiation shields, good thermal breaks and thermal isolation, and low-emissivity materials.

Some low-emissivity schemes include silver and gold plating, but polished copper potentially has comparably low emissivity. The operational challenge of using polished copper is to keep the copper from oxidizing or otherwise tarnishing and thus increasing the emissivity. To this end, a copper passivation technique was developed at PNNL (Hoppe et al. 2007) that appears to work quite well, but the resulting emissivity of the cleaned copper was not known. Thus, the secondary objective of this project was to measure the emissivity of the PNNL-passivated copper.

Experience from accelerator-based cryostat design for superconducting magnets (see, for example, Bourcey et al. 2002) and from good HPGe cryostat designs (see, for example, Becker et al. 2003) suggests that changing several aspects of conventional designs of germanium detectors and their cryostats could prove beneficial. While commercial germanium detectors and cryostats are available, they are better suited to the laboratory, where LN-cooled systems with small cryostats can be filled every 12 hours or so, and there are no space or weight limitations. Larger cryostats are available for single detectors but 
an array of detectors using larger cryostats would be bulky and be inappropriate for space-limited applications. Mechanically cooled systems require less attention and are somewhat compact but require a fair amount of electrical power to operate. Another feature of commercially available systems is that they typically use multi-layered insulation, consisting of aluminized-mylar "superinsulation" sheets. It is difficult to fabricate a compact system that does not suffer from thermal shorts in this fashion (Bourcey et al. 2002). Cryostats in superconducting magnets typically use "grounded" (LN-cooled) radiation shields to minimize liquid helium boil-off. Using a grounded shield at an intermediate temperature cooled by a Peltier cooler has been done successfully for HPGe detectors (Becker et al. 2003), but requires a more complicated design to sink the heat from the cooler. The concept of a single "floating" radiation shield is much simpler to build and requires no maintenance.

Thus, it was the goal of this project to design a system that, using the above techniques, could outperform commercially available systems by operating for many days on a single charge of about 10 liters of LN, while (1) minimizing its size and the amount of LN required, (2) not requiring any electrical power for cooling, and (3) still using commercially available components. Specifically, the goal was to design a cryostat that would house a highly-efficient, compact array of 14 germanium detectors in a small vessel that would draw less than 5 watts power. This report presents the design and testing of a cryostat that meets these specifications.

\section{Cryostat Design and Experimentation}

The test cryostat is shown in Figure 1. The cryostat consists of three parts: a stainless steel outer vacuum vessel, a copper floating radiation shield, and an aluminum inner LN dewar. The entire cryostat was designed for an anticipated "payload" of $1460 \%$ HPGe detectors, configured in two layers of seven detectors each. The initial cooling will be done conductively by a thermal short. The detectors will be cooled radiatively by the inner LN dewar during deployment. The operating power draw $(\sim 100 \mathrm{~s}$ of $\mu \mathrm{W})$ of the germanium crystals is small enough to allow this mode of operation.

\section{Outer Cryostat Wall}

The outer cryostat wall was a stainless steel vacuum vessel 24 inches in diameter. To minimize the emissivity of the outer wall, the inside of the wall was covered with two 1/32-in.-thick passivated copper sheets. Each sheet was rolled into a semi-cylinder "D" shape that just fit into the cylindrical stainless steel vessel. The bottom cap was lined with a 1/32-in.-thick sheet of passivated copper. The top cap, due to the many ports, had copper foil covering most of the flat part of the cap. The base pressure achieved in the vessel was $3 \times 10^{-6}$ Torr. The entire cryostat was weighed throughout testing to measure the LN boiloff rate. 


\section{Radiation Shield}

The floating shield design was thought superior to the multi-layered insulation (MLI) design because of the compactness requirements, the inevitable thermal shorts that accompany MLI systems, and the poor vacuum performance of MLI.

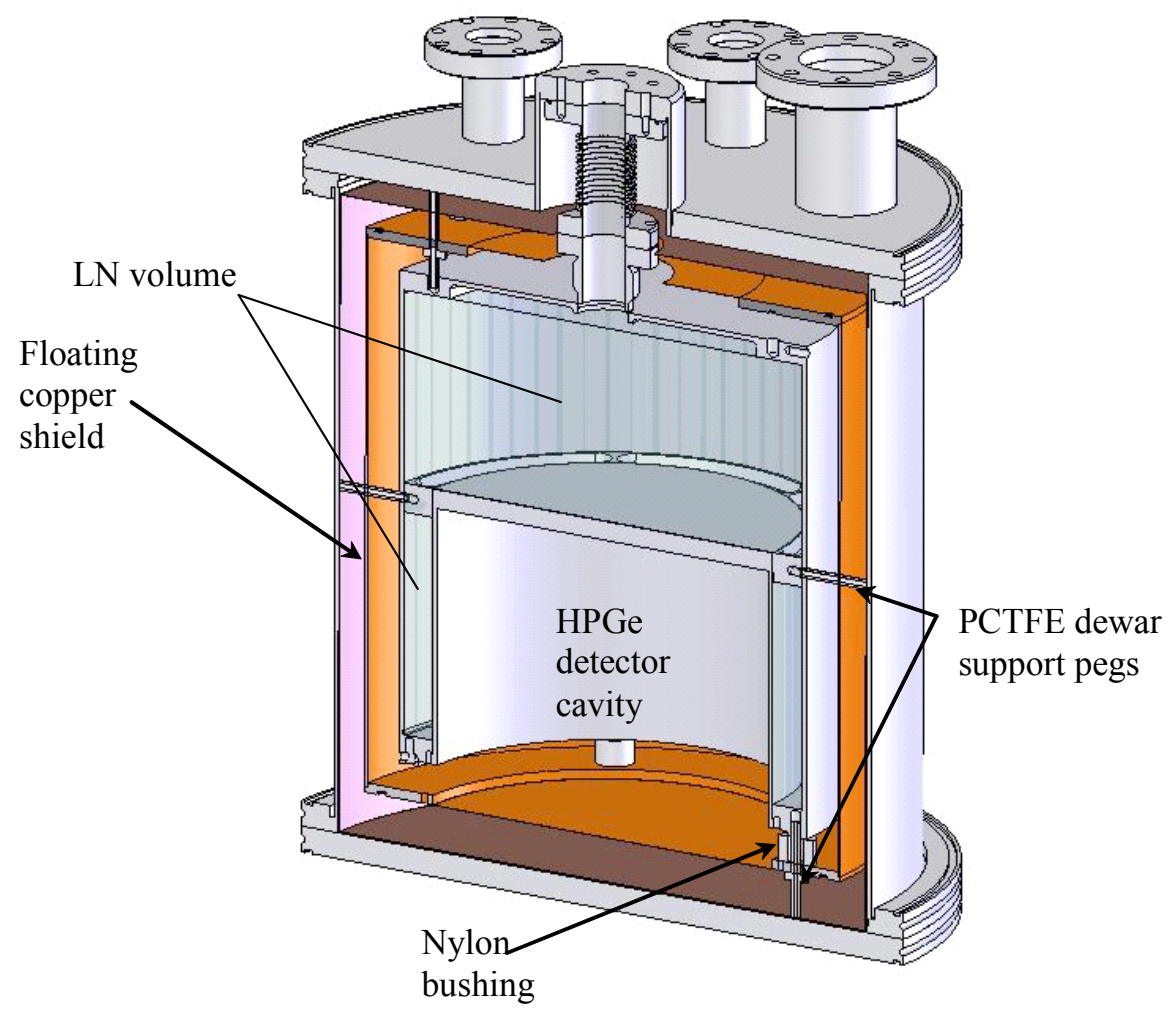

Figure 1. Schematic of the cryostat as tested in the lowest power configuration. Notice the copper lining on the inside of the outer walls, and the copper foil window at the bottom of the radiation shield.

Sides: The radiation shield consisted of two semi-cylindrical sheets of 1/32-in.-thick copper. The radius of curvature was such that the copper was halfway between the inner diameter of the outer cryostat wall and the outer diameter of the inner aluminum dewar.

Ends: The top and bottom part of the radiation shield consisted of two stainless steel annuli. Both annuli were wrapped in copper foil to minimize direct radiation paths from the outer cryostat to the inner dewar. An additional sheet of copper foil was attached to the bottom annulus which completed the closed cylindrical radiation shield.

The radiation shield was isolated from the outer cryostat by polychlorotetrafluoroethylene (PCTFE) standoff legs on both top and bottom of the dewar. Similar legs were attached on the cylindrical surface 
of the dewar to maintain the distance between the radiation shield and the dewar. These legs also serve to keep the inner assembly of dewar plus radiation shield from touching the outer cryostat wall during mobile operation and tilting of the spectrometer. To immobilize the annuli to facilitate ease of handling during assembly and disassembly of the cryostat, additional nylon standoffs were used on the bottom of the annulus.

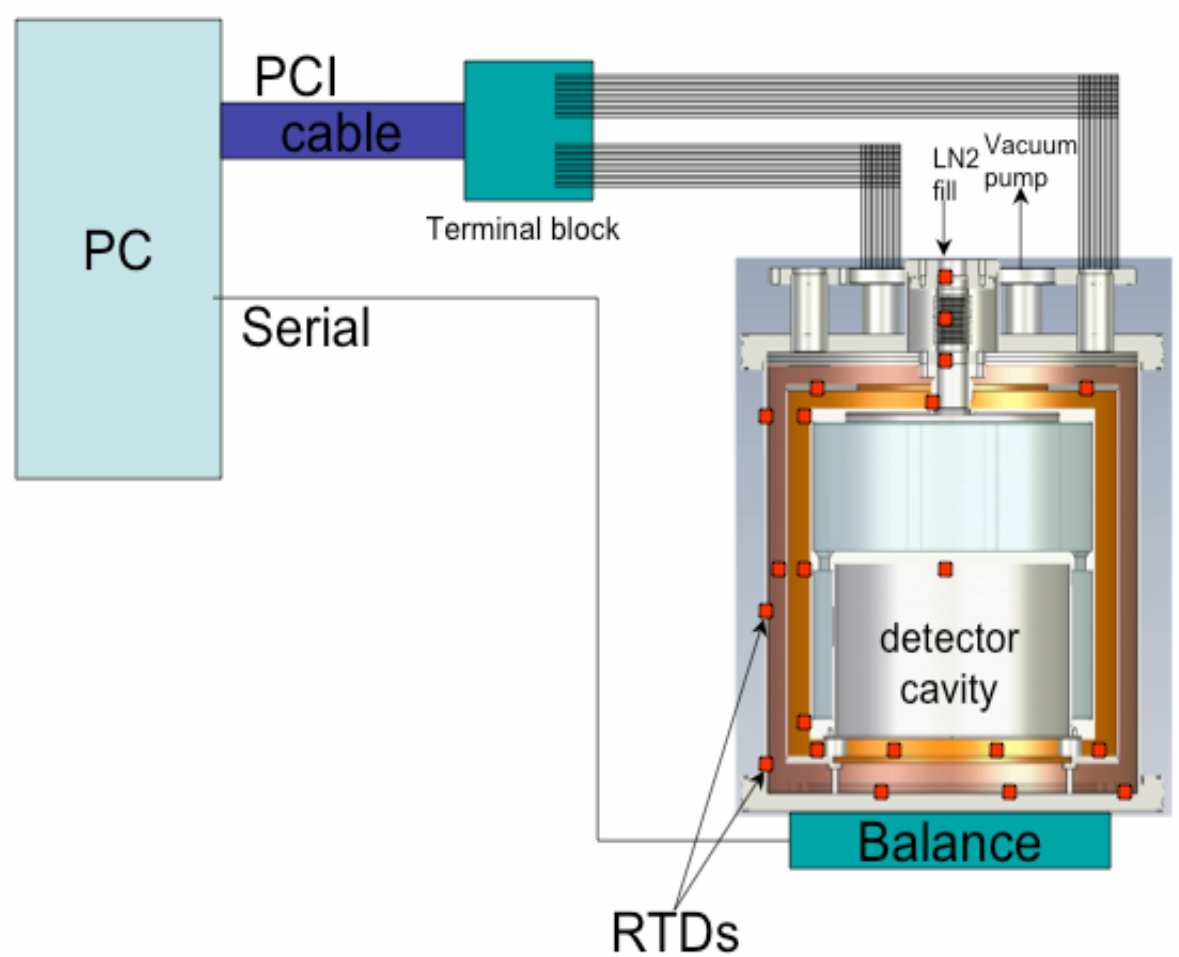

Figure 2. Schematic of the measurement setup. RTDs were placed throughout the inside of the cryostat and temperatures were recorded every 100 milliseconds. The weight of the cryostat vessel was logged throughout the measurement and was used to determine the LN boil-off rate. The data was taken through the PCI and serial buses of the computer.

\section{Dewar}

The dewar was constructed as a can with a recessed bottom half that housed the HPGe spectrometer. The can was carefully designed to allow the LN to surround the crystals on three sides, allowing the LN to fill the "skirt" around the germanium elements. The dewar was constructed entirely out of aluminum, including the welds. The dewar holds eight liters of LN. 


\section{Dewar Neck}

A stainless steel neck and flange was explosion-welded to the top of the aluminum dewar. During vacuum leak tests, Vacseal ${ }^{\mathrm{TM}}$ was used to seal the remaining leaks. A thin-walled stainless steel bellows provided the vacuum seal. This seal minimized conductive heat load through the neck because stainless steel is a relatively poor conductor. Vacuum seals were made with buna-n o-rings. Since the o-rings did not come in contact with cold surfaces, they did not leak during the test.

\section{Copper Passivation}

Each sheet of copper in the outer wall of the cryostat was passivated using PNNL-developed techniques (Hoppe et al. 2007) of sequential dilute sulfuric and citric acid baths. The primary objectives of the passivation are to reduce the oxide layers and minimize/preserve the emissivity of the copper by preventing further oxidation/tarnishing. The cylindrical halves of the radiation shield were also passivated in this manner.

\section{Temperature Measurement Apparatus}

Temperatures throughout the cryostat were measured using 24, 1000-ohm platinum RTD sensors (part no. F3142 from Omega). These RTDs conform to the European DIN 43760 standards, class B tolerance specifications (the temperature-dependent measurement uncertainty is $\pm[0.3+0.005|\mathrm{~T}|]^{\circ} \mathrm{C}$ ). The RTDs were attached to the various surfaces by first applying a bead of Apiezon NTM low-vapor pressure grease (which is solidified at $77 \mathrm{~K}$ ) then copper-taping the RTDs in place. This attachment technique worked for all but one location in the cryostat.

The RTDs chosen for the test were 1000-ohm to minimize uncertainties and systematic errors from lead-wire resistance variations. Four-wire readout was employed to minimize the systematic temperature offset error arising from the excitation current flowing through the measurement leads. The leads to the RTDs were kapton-coated 30-AWG copper wire. The leads were various lengths ranging from 1 to 4 feet long depending on the placement of the RTDs.

The RTDs were read-out by a National Instruments PCI-4351 PCI card installed in a PC. The card could accommodate 14 channels at one time. Because there were 24 RTDs, two separate measurements were taken for each geometric configuration.

\section{Analysis and Results}

An initial cool-down and warm-up cycle was carried out to measure the heat load without the floating radiation shield present and to test the measurement system. The heat load was determined to be 13 watts, as measured by LN boil-off rate, mostly from the large radiative heat transfer from the roomtemperature walls to the LN-temperature dewar. The measurement confirms the low conductive heat load on the dewar, through the bellows and through the legs. 
A second measurement was performed with the shield cylindrical wall present but the end caps absent. This test resulted in a heat load of roughly 6 watts (compared to 13 watts before), because of the larger radiative heat load present from the room temperature wall shining directly onto the dewar.

A third measurement was performed with the entire shield intact, yielding an overall average heat load of 4.25 watts, and a baseline heat load (after initial cool-down) of 4.07 watts. The results of these measurements will be discussed in more detail below.

\section{Heat Load Sources}

As constructed, the measured heat load was assumed to be due to infrared radiation from the shield and conduction to the shield and the outer cryostat from the mounting hardware. To make accurate emissivity measurements, the conduction paths must be properly identified and accurately quantified. The identified conduction paths were the plastic (PCTFE and nylon) standoffs, the dewar neck (bellows) and the RTD leads. The RTD leads contributed $\leq 70 \mathrm{~mW}$ of conductive heat load. The bellows contribute approximately 0.3 watts. The dominant conduction paths were determined to be the legs of the dewar. These legs provided a calculated 1.2 watts of conductive heat load, mostly along the path from the radiation shield to the dewar. The total conductive heat load was 1.3(3) watts. The heat load uncertainty is primarily due to the uncertainty in the thermal conductivity of the nylon and PCTFE used for the standoffs. Because we did not measure the thermal conductivity of the materials used directly, the range of values were extracted from literature and sets the uncertainty band.

\section{Temperature Features of the Data}

On the bottom annulus three holes, separated by $120^{\circ}$, allow the standoff legs to pass through the annulus and rest on the bottom of the vacuum vessel. Small ridges on the legs support the annulus between the dewar and the outer wall of the cryostat. The temperature of the bottom annulus recorded near the legs was $1{ }^{\circ} \mathrm{C}$ higher than between the legs. This data is evidence for the fairly high heat load (nearly 0.5 watt) created by the additional spacers around the standoff legs. The RTDs mounted on the top annulus, where no nylon bushings were present, did not exhibit this behavior. The cool down time for the bottom annulus (4.1 hours) of the heat shield was half as long as that of the top annulus ( 8.2 hours), consistent with the different masses of the two annuli.

\section{Emissivity Measurements}

The average LN boil-off rate was used to determine heat load on the cold mass, and this measurement was used to determine the emissivity of the copper used for the radiation shield and used in the cryostat walls. Three pieces of the radiation shield existed: the cylindrical part (1/32-in.-thick sheet), and the two end caps, which overlapped the ends by about 4 inches. The cylindrical part was passivated using the PNNL-developed method. 


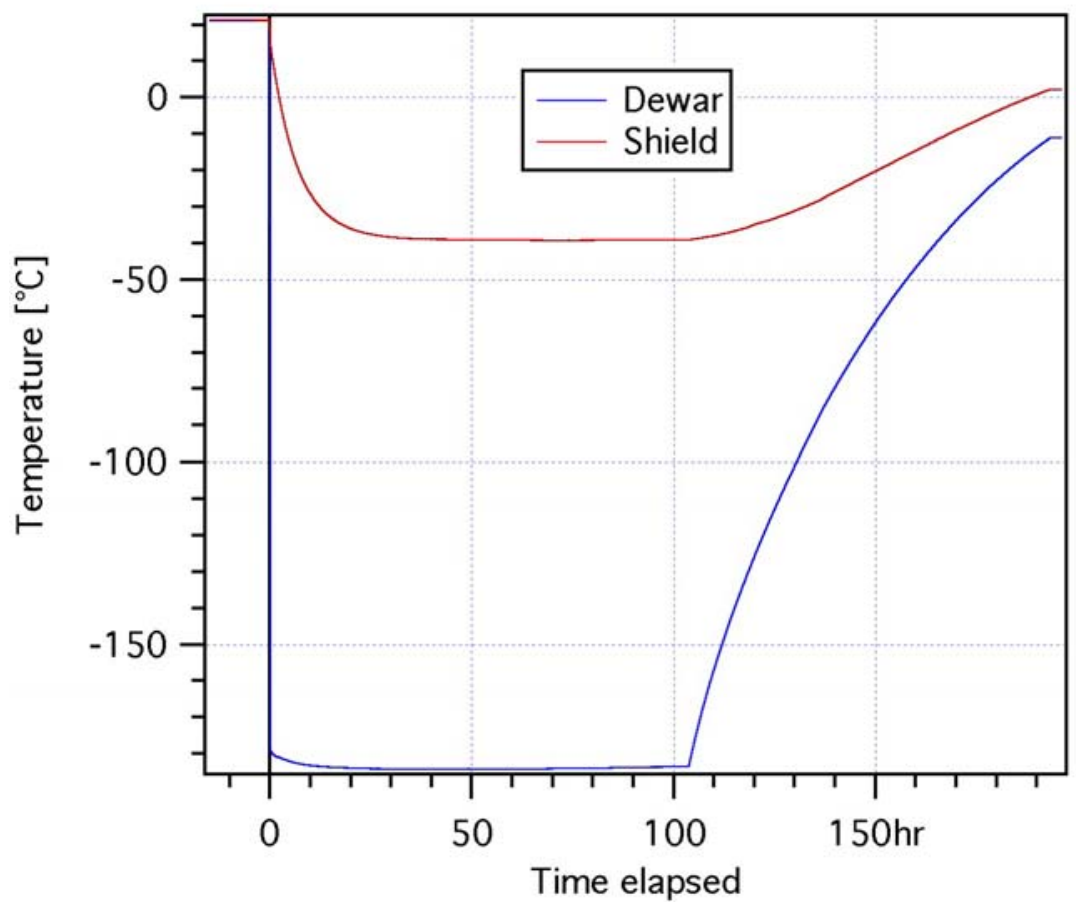

Figure 3. Temperature profile of the dewar and radiation shield during one of the tests. Note that the bottom and top end caps were not present for this data set, resulting in a higher heat load and shorter hold time.

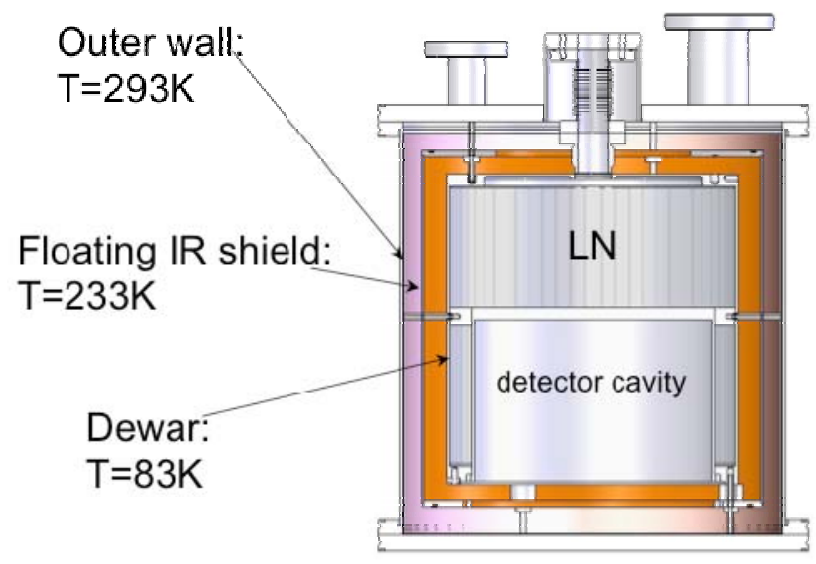

Figure 4. Measured equilibrium temperatures with a full loading of LN 


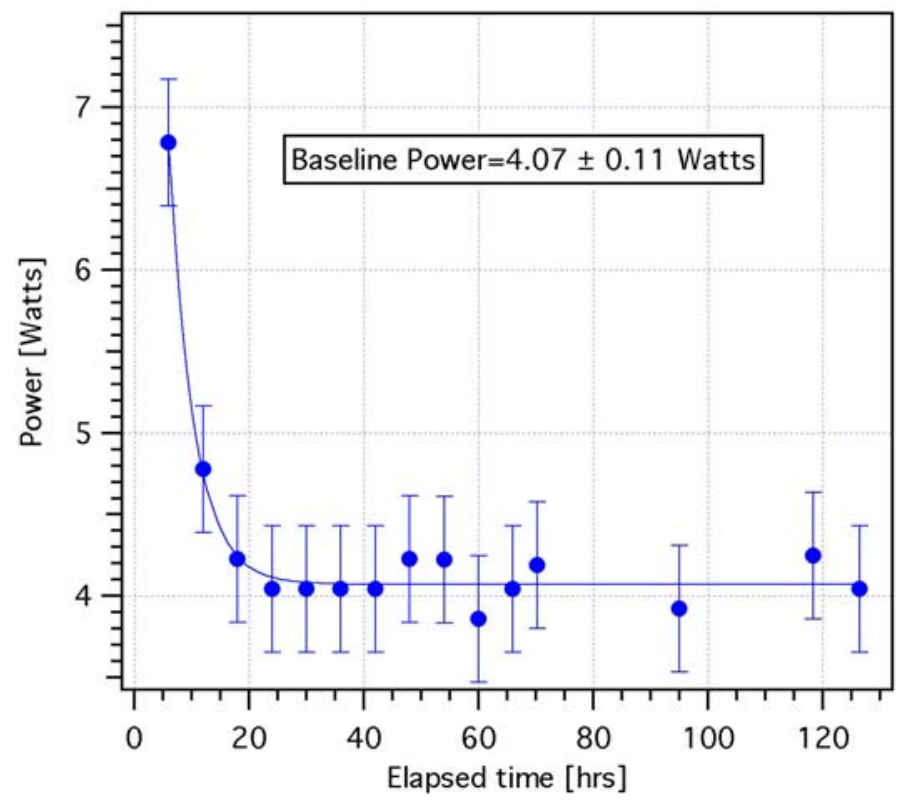

Figure 5. Instantaneous power vs. elapsed time. From a single-exponential fit, the baseline power is $4.07 \pm 0.11$ Watts and the decay constant is $4.3 \pm 1.7$ hours.

The analysis performed to determine the emissivity follows closely what was done in a previous report (PNL-16378). The general prescription is as follows: The total thermal power being transferred to the cold mass, $P_{\text {tot }}$ is measured by the $\mathrm{LN}$ boil off rate. This total power consists of two parts: conductive power, $P_{c o n}$, i.e. heat transferred directly through the supporting legs and standoffs, and radiative power, $P_{\text {rad }}$, i.e. head radiated directly from the copper radiation shield to the aluminum dewar. Thus the total heat load on the dewar can be expressed as $P_{\text {tot }}=P_{c o n}+P_{\text {rad }}$. Because the pressure in the vessel was $3 \times 10^{-6}$ Torr, convective heat transfer was negligible. Conduction of heat through a material with thermal conductivity $k$, length $d$, and cross sectional area $A$ between two temperatures $T_{1}$ and $T_{2}$ is given by $P=\frac{k A\left(T_{2}-T_{1}\right)}{d}$. The conductive portion of the total heat load is then deduced by summing over all conductive paths to the cold mass:

$$
P_{\text {con }}=\sum_{i=1}^{N} \frac{k_{i} A_{i}\left(T_{2}^{i}-T_{1}^{i}\right)}{d_{i}} .
$$

The conductive portion is then subtracted from the total heat load; the remainder is the radiative portion. The radiative heat load depends on the temperatures of the concentric surfaces (the dewar and the radiation shield), their surface areas, their emissivities and to a lesser extent their shape. For a closed concentric geometry, such as the dewar cylinder surrounded by the radiation shield, the radiative heat load can be approximated as follows: 


$$
P_{r a d}=\frac{\sigma A_{1}\left(T_{1}^{4}-T_{2}^{4}\right)}{\frac{1}{\varepsilon_{1}}+\left(\frac{1}{\varepsilon_{2}}-1\right)\left(\frac{A_{1}}{A_{2}}\right)}
$$

where $\sigma$ is the Stefan-Boltzmann constant, $A_{1}$ and $A_{2}$ are the areas of the cold and warm surfaces, and $\varepsilon_{1}$ and $\varepsilon_{2}$ are the emissivities of the surfaces. Since the only unknown in the equation is the emissivity of the copper, the emissivity of the copper is easily extracted using the other (known or measured) quantities.

The thermal conductivity of the standoff materials was not measured, so literature values were used for these materials. The additional standoff spacers were made from nylon (polyamide) and can have a thermal conductivity ranging from $k=0.25$ to $0.5 \mathrm{~W} / \mathrm{m}-\mathrm{K}$. Marquardt et al. (2000) present a value of $0.35 \mathrm{~W} / \mathrm{m}-\mathrm{K}$ which only varies by $10 \%$ from room temperature down to $80 \mathrm{~K}$; that value was used in these calculations. The standoffs were made of PCTFE $(k=0.25(5))$. Using these numbers, the calculated conductive load is 1.3(3) Watts. This value, combined with the baseline power consumption (measured by LN boil-off) of 4.07(11) watts yields an emissivity of $2.6(3) \%$ for the passivated copper, using an emissivity for the aluminum dewar of $10(2) \%$. The uncertainty in the measured emissivity of the passivated copper is set by the uncertainty in the thermal conductivity of the materials and the uncertainty in the emissivity of the aluminum dewar. This result agrees well with the result presented in PNL-16378 of $2.5(1.5) \%$.

\section{Future Work}

Based on the results presented here a prototype has been designed that incorporates most of the cryogenic features of this cryogenic test configuration. The prototype uses only passivated copper but may benefit from using lower emissivity (1-2\%) materials such as silver or gold, similar to what was done in Becker et al. (2003). The prototype detailed design is finished and it is intended that it will be constructed and tested in fiscal year 2008.

\section{Summary}

A low-power cryostat based on a floating radiation shield was designed and constructed. A demonstrated power consumption of 4.07(11) watts was measured. The emissivity of the passivated copper was measured to be $2.5(3) \%$. A germanium spectrometer installed in this cryostat, once cooled, would remain cold for 5.5 days with a single loading of 8 liters of LN, making it useful for semi-remote deployment applications.

\section{Acknowledgments}

This work was supported by the US DOE Office of Non-Proliferation NA-22. 


\section{References}

Becker JA, CP Cork, L Fabris and NW Madden. 2003. "Portable, low-power, mechanically cooled Ge spectrometer." Nucl Instrum Meth A 505(1-2):167-169.

Bourcey N, L Nielsen, V Parma, P Rohmig, E Roy and J-B Bergot. 2002. "Thermal Performance of the LHC Short Straight Section Cryostat.” In Proceedings of EPAC 2002, pp. 2526-2528. Paris, France.

Hoppe EW, A Seifert, CE Aalseth, PP Bachelor, AR Day, DJ Edwards, TW Hossbach, KE Litke, JI McIntyre, HS Miley, SM Schulte, JE Smart and GA Warren. 2007. "Cleaning and passivation of copper surfaces to remove surface radioactivity and prevent oxide formation." Nucl Instrum Meth A 579(1):486489.

Lepel EA, BD Geelhood, WK Hensley and WM Quam. 1998. "A field-deployable, aircraft-mounted sensor for the environmental survey of radionuclides." J Radioanal Nucl Ch 233(1-2):211-215.

Marquardt ED, JP Le and R Radebaugh. 2000. "Cryogenic Material Properties Database." Presented at the 11th International Cryocooler Conference. June 20-22, 2000. Keystone, Colorado.

Fast JE, et al., 2007. “MARS Initial Cryogenic Testing”, PNL-16378. 